\title{
THE PRODUCTION OF ROTATION MEASURE GRADIENTS IN THE LOBES OF EXTRAGALACTIC RADIO SOURCES
}

\author{
G.V. BICKNELL, R.A. CAMERON \\ Mount Stromlo and Siding Spring Observatories \\ Institute of Advanced Studies, Australian National University \\ R.A. GINGOLD \\ Australian National University Supercomputer Facility
}

\begin{abstract}
It is proposed that the large rotation measure gradients observed in the lobes of some extragalactic radio sources are produced by non-linear Kelvin-Helmholtz surface waves. Related small scale turbulence is responsible for a "diffuse spray" of relativistic plasma and magnetic field into the surrounding medium. The mixture of magnetic field and thermal plasma causes large rotation measure variations on the scale of the Kelvin-Helmholtz waves. An order of magnitude prediction of the effect is in good agreement with the observations of Cygnus A and PKS 2104$25 \mathrm{~N}$.
\end{abstract}

\section{Introduction}

Some extragalactic radio sources exhibit prominent bands of rotation measure across various parts of their lobes. In Cygnus A (Dreher, Carilli and Perley 1987), excursions in rotation measure of the order of $1000-4000 \mathrm{rad} \mathrm{m}^{-2}$ have been observed. The radio source PKS 2104-25 (Cameron 1989) also exhibits smaller but significant rotation measure gradients of the order of $100 \mathrm{rad} \mathrm{m}^{-2}$. Our view of these two sources is that the rotation measure variations differ in degree rather than in kind and that they are related to the turbulent mixing of magnetic field from the lobe into the surrounding medium through the non-linear development of the Kelvin-Helmholtz instability. In this paper, we discuss some of the pertinent data, present some results of work on simulation of the Kelvin-Helmholtz instability and then discuss the mechanism by which the rotation measure bands may be produced.

\section{Some features of the Cygnus A and PKS 2104-25 data}

At least some of the rotation measure features in Cygnus A (DCP) can be associated with surface waves. Regions 1 and 3 and 4 in the left panel of figure 1 seem to be waves on the surface of the lobe. In fact regions 3 and 4 seem to be a train of waves. Less certain surface waves are the regions marked 2 and 5 in figure 1 . It is significant that these waves 


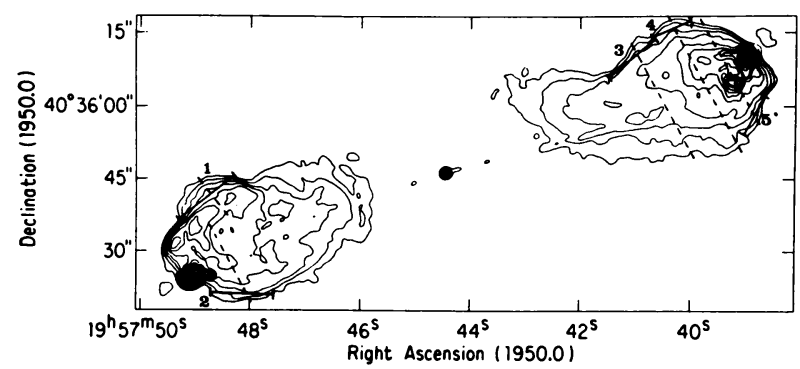

Figure 1. Left panel: Contour map of Cygnus A from DCP with the possible regions of surface waves indicated. Right panel: the corresponding rotation measure contour map. Both maps reproduced by permission R. A. Perley.

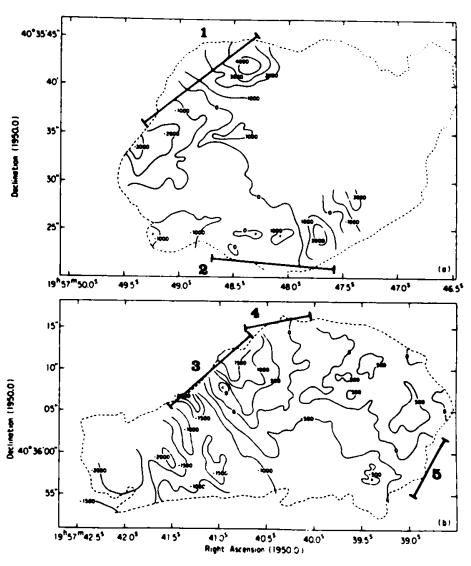

are adjacent to regions of significant rotation measure variation shown in the right panel of figure 1 .

The radio source PKS 2104-25 N (see BCG) also exhibits a prominent surface wave on the northern boundary of its northern lobe. The grey-scale rotation measure image at 13 " resolution shows a prominent band of rotation measure across the lobe. The RM in this band varies from 0 to $\sim 120 \mathrm{rad} \mathrm{m}^{-2}$ and back again.

\section{3D Simulations of the Kelvin-Helmholtz Instability}

The theory presented here was based upon computational work by Bicknell and Gingold (1989) on the three dimensional evolution of the Kelvin-Helmholtz instability, using a vectorized version of Smoothed Particle Hydrodynamics (SPH) adapted for Large Eddy Simulations (Bicknell (1989)). The streams in this simulation are of equal density, have Mach numbers of 1.0 (lower stream) and 0.1 (upper stream). The streams were perturbed by a multi-frequency, random-phase perturbation.

Figure 2. A snapshot from a 3D numerical simulation of the KelvinHelmholtz instability. The greyscale image is produced by assigning a "colour" of \pm 1 to the particles in the two streams

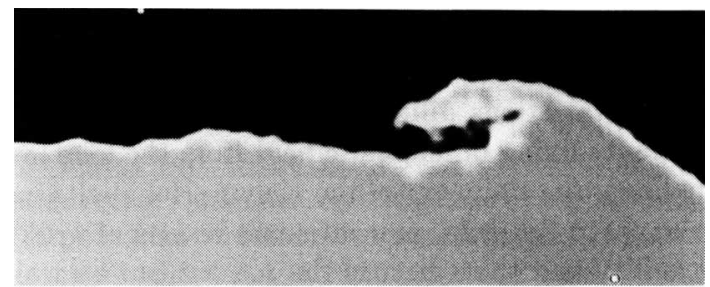

The striking result which is evident in figure 3 is the ultimate growth of large scale structure due to the merging of high wave number waves in a sort of inverse cascade. Indeed, linear Kelvin-Helmholtz waves grow at a rate which is proportional to the wave number and if the merging of small scale waves were not to occur, then the late-time evolution of the $\mathrm{KH}$ instability would be dominated by high wave numbers.

\section{Turbulent Diffusion of Particles and Magnetic Field}

The lobe/IGM interface is severely convoluted by the evolution of the non-linear KH waves and some small-scale turbulence is produced at the lobe/IGM interface. However, 
it must be remarked that the turbulence is not fully developed. Related to the turbulence, there is an associated diffusion of relativistic particles and magnetic field into the intergalactic medium. This does not result in substantial diffusion but rather in a fine "spray" of particles and field surrounding the lobe. As shown below, this spray can be sufficient to cause the observed rotation measure variation.

Following Parker (1979), we estimate the diffusion rates as follows. The ensembleaveraged values for relativistic electron number density, $n_{\text {rel }}$, and magnetic field, $B^{\alpha}$, are given by:

$$
\left\langle\frac{n_{\text {rel }}}{n_{b}}\left(\mathbf{x}, t_{2}\right)\right\rangle=\left\langle\frac{n_{\text {rel }}}{n_{b}}\left(\mathbf{X}, t_{1}\right)\right\rangle \quad\left\langle\frac{B^{\alpha}}{n_{b}}\left(\mathbf{x}, t_{2}\right)\right\rangle=\left\langle\frac{B^{\beta}}{n_{b}}\left(\mathbf{X}, t_{1}\right) \frac{\partial x^{\alpha}}{\partial X^{\beta}}\right\rangle
$$

where $n_{b}$ is the baryon density and $\mathbf{x}$ and $\mathrm{X}$ are the initial and final coordinates, at times $t_{1}$ and $t_{2}$ respectively, of an element of fluid.

Invoking Parker's "amnesia hypothesis" for the ensemble average of the accumulated strain of a fluid element, implies that $n_{\text {rel }} / n_{b}$ and $B^{\alpha} / n_{b}$ diffuse similarly so that the profiles of $B^{\alpha}$ and $n_{\text {rel }}$ in the mixing region are similar as well.

For order of magnitude purposes, we can estimate the amount of diffusion by approximating the convoluted lobe/IGM interface by a locally plane shear layer. In view of the above, the diffusion of a scalar field with a diffusion coefficient $\eta_{T} \approx 0.4 v_{T} l_{T}$, can be used to represent both the diffusion of $n_{\text {rel }} / n_{b}$ and $B^{\alpha} / n_{b}$ to give a diffusion length: $l_{\mathrm{diff}} / z=0.6\left(v_{T} / V\right)^{1 / 2}\left(l_{T} / z\right)^{1 / 2}$ where $z$ is along the shear layer, $x$ is perpendicular to it and $V$ is the relative speed of the lobe and IGM. The separation between waves increases linearly with distance from $z=0$ and so also does $l_{T}$ since the scale of turbulence should scale with the structures which are driving it. For fairly small values, $v_{T} / V \approx l_{T} / z \sim 0.1$, $l_{\mathrm{diff}} / z \approx 0.06$. This diffusive width should be compared to the crest to trough depth of each of the $\mathrm{KH}$ waves, $l_{\mathrm{KH}} \approx 0.3 \lambda$ where $\lambda$ is the wavelength which itself scales with $z$ due to the merging of waves. The smaller diffusive width indicates that the values of magnetic field and relativistic particle density may be a factor of a few less than the corresponding values in the lobe.

Since the turbulence is small scale the large scale structure is driven by the large scale velocity field and the field oscillates on the scale of the large scale $\mathrm{KH}$ waves.

\section{The Rotation Measure Variation}

Virtually all of the diffused magnetic field will exist within a distance of the lobe/IGM interface equal to the crest-trough distance, $d_{\mathrm{KH}}$, which is the relevant path length to use for an estimate of the rotation measure. From the simulations (and also from Brown and Roshko (1974)) $d_{\mathrm{KH}} \approx \lambda_{\mathrm{KH}}$ where $\lambda_{\mathrm{KH}}$ is the (increasing) wavelength between crests. Hence the following estimate of the rotation measure excursion over a wavelength:

$$
\Delta \phi \approx 490\left(\frac{n_{\text {mix }}}{n_{\mathrm{cl}}}\right)\left(\frac{B_{\text {mix }}}{B_{\text {lobe }}}\right)\left(\frac{n_{\mathrm{cl}}}{\mathrm{cm}^{-3}}\right)\left(\frac{B_{\text {lobe }}}{\mu G}\right)\left(\frac{\lambda_{\mathrm{KH}}}{\mathrm{kpc}}\right) \mathrm{rad} \mathrm{m}^{-2}
$$

where $n$ refers to the thermal number density and the subscripts mix and cl refer to the diffusive mixing region and the cluster respectively. $\Delta \phi_{\text {fid }}$, corresponding to $B_{\text {mix }} / B_{\text {lobe }}=$ 


\begin{tabular}{|c|c|c|c|c|c|}
\hline \multicolumn{6}{|c|}{ Comparison of Rotation Measure Estimates with Data } \\
\hline Region & $\begin{array}{c}B_{\mathrm{P}_{\text {min }}} \\
(\mu G)\end{array}$ & $\begin{array}{c}n_{\mathbf{e}} \\
10^{-3} \mathrm{~cm}^{-3}\end{array}$ & $\begin{array}{c}\lambda_{\mathbf{e}} \\
\mathrm{kpc}\end{array}$ & $\begin{array}{c}\Delta \phi_{\text {fid }} \\
\mathrm{rad} \mathrm{m}^{-2}\end{array}$ & $\begin{array}{c}\Delta \phi_{\text {obs }} \\
\mathrm{rad} \mathrm{m}^{-2}\end{array}$ \\
\hline Cygnus A -1 & 64 & 6 & 24 & 4500 & 7000 \\
Cygnus A - & 38 & 6 & 21 & 2300 & 3000 \\
Cygnus A - 3 & 35 & 6 & 20 & 2100 & 3500 \\
Cygnus A - & 41 & 6 & 14 & 1700 & 1000 \\
Cygnus A - 5 & 51 & 6 & 15 & 2300 & 1500 \\
\hline PKS 2104-25 N & 8 & 1.5 & 15 & 135 & 160 \\
\hline
\end{tabular}

$n_{\text {mix }} / n_{\mathrm{cl}}=1$ is tabulated for the various regions of Cygnus $\mathrm{A}$, and the northern lobe of PKS 2104-25 N.

The values of $\Delta \phi_{\text {fid }}$ compare quite favourably with the observed values. Furthermore, because of the bow shock surrounding the lobe, the thermal density in the immediate vicinity of the lobe is a factor of 2-4 higher and the requirement on the strength of the magnetic field is weakened to $B_{\text {mix }} / B_{\text {lobe }}$ as implied by the above discussion on diffusion.

\section{Depolarization}

This mechanism need not lead to significant depolarization. The Faraday depolarization is so large in the mixing region that it contributes nothing to the polarized flux at either 6 or $20 \mathrm{~cm}$., say and the percentage polarization does not change as a function of wavelength. Moreover, the emissivity in the mixing region $\epsilon_{\nu, \text { mix }} \propto P_{\text {rel }} B^{3 / 2} \propto n_{\text {rel }}^{4 / 3} B^{3 / 2}$ so that $\epsilon_{\nu, \text { mix }}=\epsilon_{\nu, \text { lobe }} \times\left(B_{\text {mix }} / B_{\text {lobe }}\right)^{17 / 6}$ the last relationship following from the similar diffusion of particles and field. For values $B_{\text {mix }} / B_{\text {lobe }}=0.1,0.25,0.5$, the emissivity of the mixing region is $0.0015,0.02$ and 0.14 times that of the lobe respectively. This combined with the comparatively small extent of the mixing region means that in most cases it should be invisible, making the depolarizing effect of this region difficult to detect.

This theory explains several features of the observed rotation measure variations: the orientation of the normal to the bands (in the direction of the lobe backflow), their wavelength (related to Kelvin-Helmholtz surface waves) and the magnitude of the effect. Our order of magnitude estimate can be tested by observations of other sources wherein the wavelength of the waves can be measured from the wavelengths of the surface waves (if obvious) or the wavelengths of the rotation measure variations themselves.

\section{References}

Bicknell G.V., 1989, SIAM J. Sci. Stat. Comput., in press

Bicknell G.V., Cameron R.A. and Gingold R.A., 1989, Ap.J., in press

Cameron R.A., 1989, Ph.D. thesis, Australian National University.

Dreher J.W., Carilli C.L., and Perley R.A. 1987, Ap. J., 316, 611 (DCP)

Parker E.N., 1979, Cosmical Magnetic Fields, Clarendon Press, Oxford. 
LESCH: What is the difference between your hydrodynamic instability and the usual picture of turbulence, including cascading?

BICKNELL: The waves coalescense but you have also the cascading process.

CONWAY: Your mechanism of explaining these high rotation measures $>1000 \mathrm{rad} / \mathrm{m}^{2}$ in terms of the ambient $n_{e}$ and the internal field $B$ is very appealing. It would seem that high RM should go with high luminosity (strictly, with high surface brightness $T_{b}$ ) which accords with known correlations. However, we should note that these RMs are a factor 100-1000 x normal values, whereas the internal field $B_{\mathbf{e q}}$ only varies by a factor 10 to 30 at most.

BICKNELL: The important factors are the cluster density which is higher in so-called "cooling flow" clusters, the lobe magnetic field which scales with the luminosity of the source and the wavelengths of the KelvinHelmholtz waves which scale with the size of the source. It is all these factors together which contribute to the very large rotation measures observed in sources such as Cygnus A.

ZANINETTI: Did you make comparison of the linear growth as given by the Kelvin-Helmholtz instabilities with your numerical simulations?

BICKNELL: There are actually two time scales involved. The first is the time scale for linear growth and is given by the usual expressions for the Kelvin-Helmholtz instability. The second is the time scale for the non-linear interactions of the waves, to be specific, the time it takes a small wave to catch up to a larger leading wave. We have not investigated in detail what this depends upon but it is certainly going to scale with the phase speeds of the linear Kelvin-Helmholtz waves.

KIM: From Perley's and your talk it seems curious that there exists a characteristic scale $\lambda$ on which a magnetic field varies. The $\lambda$ is about $20 \mathrm{kpc}$ which is obviously the size of an optical galaxy. Theoreticians have been looking for the characteristic turbulence length in an intracluster medium (ICM) which may relate to $\lambda$. In your simulation is $\lambda$ naturally coming out, or is this the result of turbulences induced in ICM by cluster galaxies with which radio lobes are interacting?

BICKNELL: In this theory the characteristic scale $\lambda$ arises from the scale of the Kelvin-Helmholtz waves on the surface of the lobe. This is determined by the non-linear interactions between waves which I have described. There is really no "natural" scale - the wavelengths increase linearly with distance from the source, in this case the hot spot at the head of the lobe. Thus, by a distance of $30 \mathrm{kpc}$, say, the wavelength is of the order of $10-20 \mathrm{kpc}$. 